\title{
Lehre digital und interaktiv gestalten
}

Im Sommer haben die Deutsche Röntgengesellschaft und die LernRad $\mathrm{GmbH}$ eine Kooperationsvereinbarung unterzeichnet, um künftig auf dem Gebiet der radiologischen Aus-, Fort- und Weiterbildung zusammenzuarbeiten. Ähnlich wie conrad, die digitale Lernplattform der DRG für die Fort- und Weiterbildung in der Radiologie, bietet auch LernRad digitale radiologische Lehrinhalte an. Wir haben Prof. Dr. Bettina Baeßler, eine der Geschäftsführerinnen der LernRad $\mathrm{GmbH}$, zur Zusammenarbeit befragt.
Prof. Dr. Baeßler, Sie haben mit Ihren beiden Kolleginnen Dr. Mareike Franke und PD Dr. Nienke Lynn Hansen die Onlineplattform „LernRad“ für digitale radiologische Lehrinhalte gegründet. Wann und wie ist die Idee zu „LernRad“ entstanden?

Lehre war für mich schon immer ein zentrales Interessengebiet - das hat schon während meines eigenen Studiums angefangen. Ich hatte das Gefühl, dass Lehre doch eigentlich auch spannender und interaktiver gehen müsste. Dieses Gefühl hat mich weiter begleitet, und während meiner Weiterbildung in Köln habe ich dann als Lehrkoordinatorin die Möglichkeit bekommen, meine Vorstellungen von guter Lehre in die Realität umzusetzen. Dabei haben sich diese Vorstellungen insbesondere im digitalen Raum nochmal weiterentwickelt, zumal ich selbst immer auf der Suche nach guten radiologischen Online-Lehrinhalten für meine eigene Weiterbildung war.

Diese habe ich aber - abgesehen von unirad, was sich aber ja primär an Studierende und nicht so sehr an Weiterbildungsassistentinnen und -assistenten richtet, und ein paar wenigen englischsprachigen Inhalten - nie gefunden; hierzulande ist die radiologische 


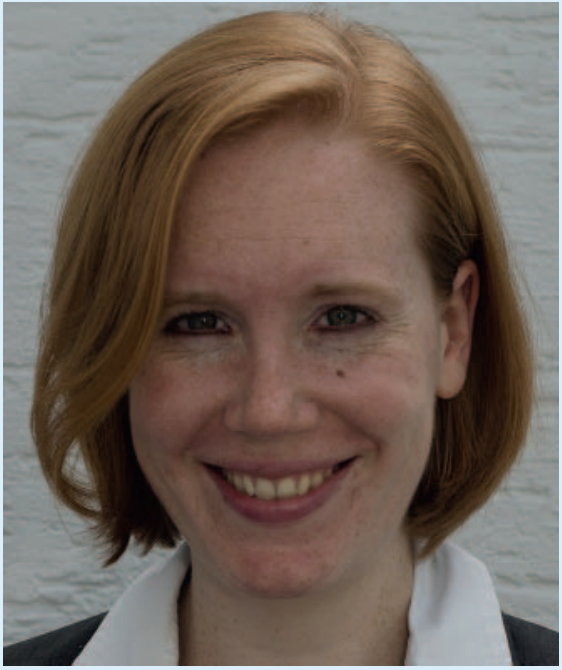

Prof. Dr. Bettina Baeßler (c) privat

Fort- und Weiterbildung immer noch sehr Dozierenden- und wenig Lernenden-zentriert und recht frontal aufgestellt. Mir persönlich hat das immer nicht so viel gebracht und ich hatte das Gefühl, dass ich all das, was mir jemand stundenlang auf irgendwelchen Standbildern gezeigt hatte, sofort wieder vergessen hatte und am echten Fall auch nicht wiederfinden würde. Dann kam die Corona-Pandemie und Nienke Hansen und ich haben gemeinsam mit einigen anderen an dem Onlinekurs CoRad-19 gearbeitet, um die radiologische Lehre deutschlandweit in vollständig digitaler Form anbieten zu können. In diesem Moment hat sich dann der schwelende Wunsch noch einmal verstärkt, eine eigene Plattform aufzubauen, die genau die Art von digitaler Lehre bietet, die wir uns vorstellen. Das ging nur über die Gründung einer Firma, denn eine solche Lernplattform erfordert natürlich auch recht viele - persönliche und finanzielle - Ressourcen.

\section{Wie sieht Ihre „Philosophie“ in Bezug auf medizinische Fortbildung aus?}

Unsere Philosophie ist die einer Lernendenzentrierten digitalen Fort- und Weiterbildung, die man sich ein bisschen vorstellen kann wie die Oberärztin oder den Oberarzt, die oder der sich neben einen setzt und einen Fall, nachdem man sich diesen erst einmal selbst in Ruhe angeschaut hat, mit aller Geduld und dem nötigen Wissen erklärt. Wir glauben, dass man am Fall durch selbstständiges, aber eben auch

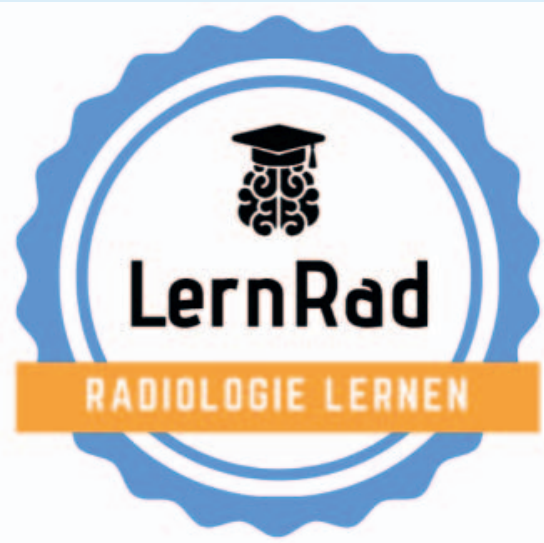

(C) LernRad-GmbH

dezent angeleitetes Lernen den besten und vor allem langfristigsten Lernerfolg hat. Hierbei sind aber nicht nur die interaktiven Dicom-Fälle mit anschließenden Video-Auflösungen entscheidend. Was uns darüber hinaus noch von anderen Anbietern abhebt, sind die interaktiven Lerninhalte, die den Lernenden helfen, sich das Wissen schrittweise selbst zu erarbeiten. Keine Frontalvorträge, sondern ein selbstständiges, angeleitetes Erarbeiten von Lerninhalten anhand von interaktiven Videos, vielfältigen Quiz und eben echten Dicom-Fällen. Die theoretischen Inhalte behält man dann, wenn man sie anschließend immer wieder an Fällen übt, deutlich besser im Gedächtnis. Wir setzen dabei auch stark auf die strukturierte Befundung und geben unseren Lernenden damit ein Werkszeug an die Hand, mit dem sie im Anschluss weiterarbeiten können. Schon Studierende lernen so in unseren Kursen, wie sie ganz systematisch an ein Bild herangehen können. So können sie das erlernte Wissen unmittelbar in ihrem klinischen Alltag einsetzen und weiter festigen.

Was hat Sie motiviert, beim Thema radiologische Fort- und Weiterbildung eine Kooperation mit der Deutschen Röntgengesellschaft einzugehen?

Wir alle sind tief in der DRG verwurzelt und waren ja auch bereits am unirad- und CoRad-19-Projekt aktiv beteiligt. Wir verstehen uns nicht als konkurrierendes Angebot zu conrad, unirad und Co., sondern möchten eine sinnvolle Ergänzung schaffen mit dem Fokus auf der Weiterbildung, weil wir hier selbst die Erfahrung gemacht haben, dass es wenige gut strukturierte Inhalte gibt. Es erschien uns deshalb nur logisch, dass wir eine Kooperation mit der DRG eingehen und haben uns sehr über die Offenheit, die uns hier entgegenschlug, gefreut. Wir denken, dass es Lerninhalte gibt, die sich auf einer rein ehrenamtlichen Basis von zwar hochengagierten, aber eben doch klinisch und anderweitig meist sehr eingespannten Kolleginnen und Kollegen nicht ähnlich hochqualitativ umsetzen lassen, wie das in einem darauf fokussierten Unternehmen der Fall ist. Hier sind als vielleicht bestes Beispiel die technischen Lerninhalte für die Facharzt-Vorbereitung zu nennen. Die Erstellung solcher Lerninhalte ist unglaublich aufwendig und zeitintensiv, wenn man sie didaktisch gut machen möchte. Das ist „so nebenbei“ und im Ehrenamt einfach nicht machbar. Genau solche Inhalte bieten wir in naher Zukunft an und ergänzen damit das bereits bestehende Angebot der DRG.

\section{Könnten Sie uns die Kooperation mit der Deutschen Röntgengesellschaft konkret beschreiben?}

Im Rahmen unserer Kooperation mit der DRG stimmen wir uns bezüglich der Kurse und Themengebiete, die wir erarbeiten, eng ab. Auf diese Weise vermeiden wir es, Redundanzen zu schaffen, sondern stattdessen wirklich eine sinnvolle Ergänzung zu den Inhalten von conrad zu sein. Unsere Kurse werden in Zukunft auf der Plattform für Weiterbildungsassistentinnen und -assistenten als Partner-Angebote angezeigt; im Gegenzug dafür bieten wir allen DRG-Mitgliedern einen Rabatt von $50 \%$ des regulären Kaufpreises auf all unsere Kurse. Somit profitieren vor allem die Mitglieder von unserer Kooperation und wir ermöglichen insbesondere den Weiterbildungsassistentinnen und -assistenten einen relativ niedrigschwelligen Zugriff auf wirklich gute Lerninhalte.

Wie sieht bei „LernRad“ die Arbeitsteilung mit lhren Kolleginnen aus?

Wir alle haben unterschiedliche fachliche Schwerpunkte, was auch mit ein Grund dafür war, dass wir uns als Trio zusammengetan haben. Frau Hansen hat ihren Schwerpunkt in der urogenitalen, vor allem der Prostatabildgebung und befindet sich derzeit in der 


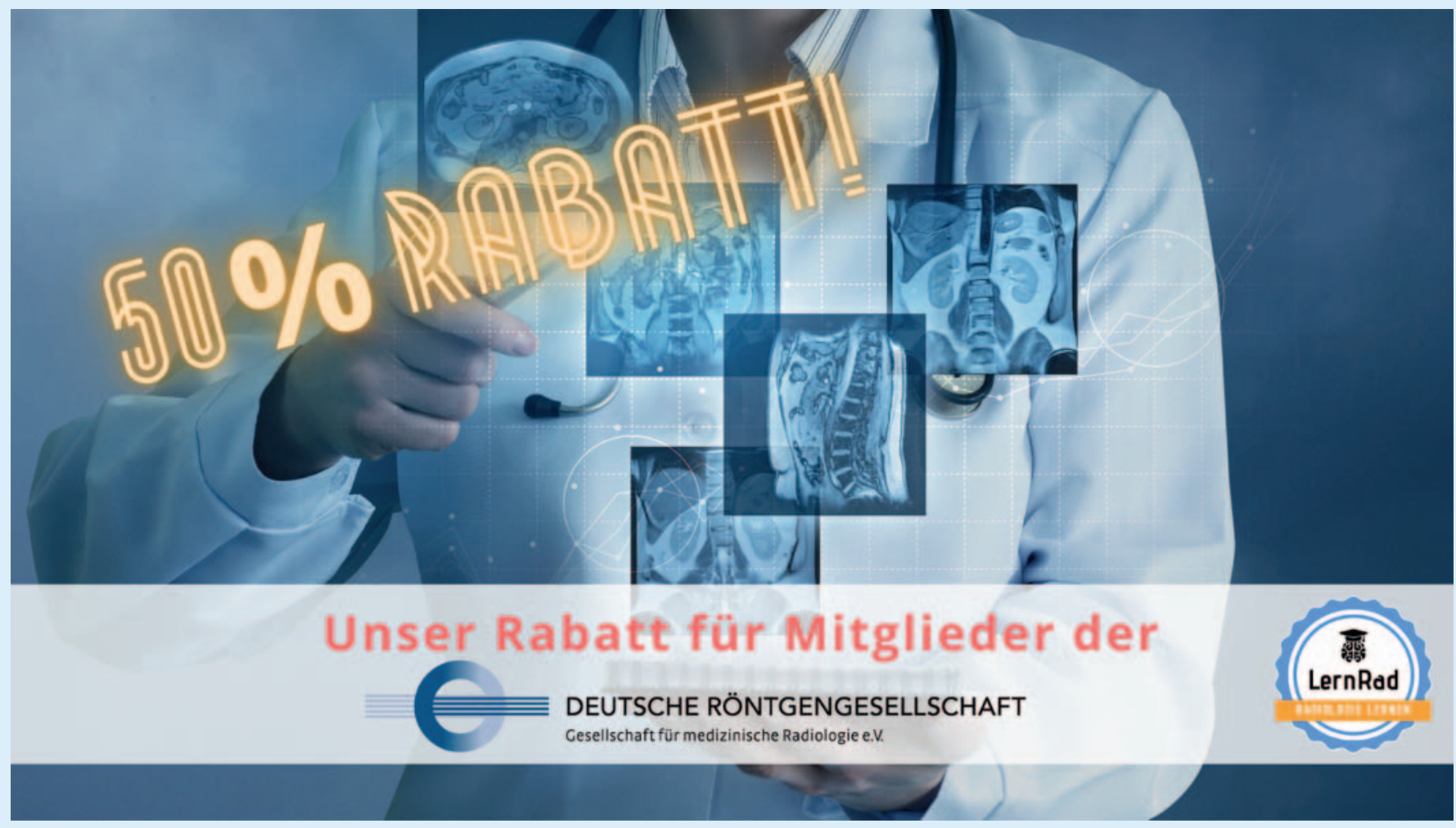

(c) LernRad GmbH

Subspezialisierung auf dem Gebiet MSK, Frau Frankes Schwerpunkte liegen im Bereich Interventionen und Abdomen- sowie Notfalldiagnostik und meine Schwerpunkte sind die kardiovaskuläre Bildgebung und die physikalisch-technischen Inhalte. Somit ergänzen wir uns fachlich schon einmal sehr gut. Entsprechend teilen wir die Kurserstellung analog unserer eigenen fachlichen Expertise auf. Alles, was wir selbst nicht abdecken können (wie z. B. die Senologie), versuchen wir in Zukunft mithilfe von externen Dozierenden abzudecken. Dozierende können also innerhalb unserer Plattform mit unserer technischen Unterstützung eigene Kurse erstellen, die sich aber streng an unseren didaktischen Maximen ausrichten müssen. Ich bin darüber hinaus noch für Marketing und Design zuständig. Generell versuchen wir aber, alle anfallenden Arbeiten paritätisch unter uns aufzuteilen.

Wie planen Sie Ihre Inhalte und welche Inhalte wollen Sie in Zukunft ausbauen?

Wir haben zunächst mit Kursen, die sich primär an Studierende in der Vorbereitung auf das M3-Examen und ganz junge Assistenzärztinnen und -ärzte richten, begonnen.
Das sind unsere Basics Kurse, mit denen wir die absoluten Grundlagen in der Thorax-, Abdomen-, Neuro- und Skelettbildgebung schaffen möchten. Von Studierenden fast aller deutschen, aber sogar auch einiger außerdeutschen Fakultäten wird dieses Angebot inzwischen bereits erfreulich gut genutzt und die Rückmeldungen sind außerordentlich positiv. Unser eigentlicher Fokus wird aber in Zukunft auf der Abdeckung des Weiterbildungscurriculums liegen, das durch das Forum Junge Radiologie in der DRG kürzlich entwickelt wurde. Hier arbeiten wir derzeit intensiv an TechnikKursen für die Vorbereitung auf die Facharztprüfung; ein erster Kurs (Röntgentechnik Teil 1) ist bereits online. Wir hatten das Gefühl, dass gerade auf diesem Gebiet ein großer Bedarf an didaktisch gut aufbereiteten Lerninhalten besteht, die im Idealfall sogar Spaß machen und nicht einfach nur ein „notwendiges Übel“ darstellen. Ich hoffe, dass es uns dadurch gelingt, nicht nur die Vorbereitung auf die technischen Prüfungsinhalte der Facharztprüfung zu erleichtern und angenehmer zu machen, sondern auch wirklich wichtiges Grundlagenwissen für die zukünftige klinische Arbeit zu vermitteln. Ich habe in meinem Alltag häufig beobachtet, dass diese Grundlagen fehlen - was unter anderem auch den interdisziplinären Austausch etwa mit dem technischen Personal oder den Medizinphysikexpertinnen und -experten nicht gerade leichter macht. Diese Lücke möchten wir schließen. Entsprechend sind unsere Technik-Kurse durchaus auch für genau diese Berufsgruppen interessant, da das Wissen, das zu den technischen Grundlagen vermittelt wird, ja überall ähnlich ist. Darüber hinaus arbeiten wir an ersten Kursen zur MSK-Bildgebung und einem AbdomenKurs für Weiterbildungsassistentinnen und -assistenten. Da auch wir 3 Gründerinnen nur im Nebenerwerb an den Kursen arbeiten, müssen wir schrittweise vorgehen und unser Angebot nach und nach erweitern.

„LernRad“ wurde von 3 Frauen gegründet. Ist das für Sie etwas Besonderes?

Primär haben wir 3 uns zusammengetan, weil wir eine ähnliche Begeisterung für gute Lehre und das Weitergeben unseres Wissens haben, und wir ergänzen uns sowohl fachlich wie auch persönlich einfach sehr gut. Nienke Lynn Hansen und Mareike Franke waren sofort begeistert, als ich 
ihnen von meiner Idee erzählt habe, und wir haben uns mehr oder weniger sofort daran gemacht, unsere eigene Firma zu gründen. Natürlich ist es in der heutigen BusinessWelt leider immer noch eine Besonderheit, wenn eine Firma von 3 Frauen geführt wird. Wir finden, dass es so etwas viel häufiger geben sollte, und möchten andere Frauen gerne dazu ermutigen, ihre Ideen ebenfalls in die Tat umzusetzen. Es gibt so viele Frauen, die gute Ideen haben, aber einfach nicht so risikobereit sind, wie vielleicht manche ihrer männlichen Pendants. Außerdem ist es für Frauen auch heute noch viel schwieriger, an Kapital zu kommen, was auch aus einigen aktuellen Studien hervorgeht. Da uns das Thema Diversität und Vielfalt bekanntermaßen sehr wichtig ist - und wir zwischen unserer Arbeit in der Diversity-Kommission der DRG und unserer Tätigkeit bei der LernRad GmbH immer wieder erstaunliche Synergieeffekte und Parallelen feststellen -, ist LernRad auch ein wenig der Versuch zu zeigen, dass eine Firmengründung auch mit Familie und Vollzeit-Klinikjob durchaus machbar ist und ein wenig mehr Diversität im Unternehmertun eine gute Sache ist. Gerade als Frauen und
Mütter sind wir durch die nicht erst durch die COVID-19-Pandemie gestiegenen Anforderungen des Alltags überhaupt erst auf die Idee gekommen, gute E-Learning-Produkte anzubieten, die zu jeder Tages- und Nachtzeit und von überall, ohne Organisation von Kinderbetreuung, Anreise und Übernachtung, genutzt werden können. Wir würden uns wünschen, dass andere unserem Beispiel folgen und die vielen weiteren guten Ideen, die in der Radiologie schon herumgeistern, ihren Weg in die Welt finden. 